\title{
COVID-19 challenge for modern medicine
}

\author{
Tomasz Dzieciatkowski ${ }^{1}$, Lukasz Szarpak $^{2}$, Krzysztof J. Filipiak ${ }^{3}$, \\ Milosz Jaguszewski ${ }^{4}$, Jerzy R. Ladny ${ }^{5}$, Jacek Smereka ${ }^{6}$ \\ ${ }^{1}$ Chair and Department of Medical Microbiology, Medical University of Warsaw, Poland \\ ${ }^{2}$ Lazarski University, Warsaw, Poland; Polish Society of Disaster Medicine, Warsaw, Poland \\ ${ }^{3} 1^{\text {st }}$ Chair and Department of Cardiology, Medical University of Warsaw, Poland \\ ${ }^{4} 1^{\text {st }}$ Department of Cardiology, Medical University of Gdansk, Poland \\ ${ }^{5}$ Clinic of Emergency Medicine, Medical University of Bialystok, Poland; \\ Polish Society of Disaster Medicine, Warsaw, Poland \\ ${ }^{6}$ Department of Emergency Medical Service, Wroclaw Medical University, Wroclaw, Poland; \\ Polish Society of Disaster Medicine, Warsaw, Poland
}

This paper was guest edited by Prof. Lukasz K. Czyżewski

\begin{abstract}
Coronaviruses cause disease in animals and people around the world. Human coronaviruses (HCoV) are mainly known to cause infections of the upper and lower respiratory tract but the symptoms may also involve the nervous and digestive systems. Since the beginning of December 2019, there has been an epidemic of SARS-CoV-2, which was originally referred to as 2019-nCoV. The most common symptoms are fever and cough, fatigue, sputum production, dyspnea, myalgia, arthralgia or sore throat, headache, nausea, vomiting or diarrhea (30\%). The best prevention is to avoid exposure. In addition, contact persons should be subjected to mandatory quarantine. COVID-19 patients should be treated in specialist centers. A significant number of patients with pneumonia require passive oxygen therapy. Non-invasive ventilation and high-flow nasal oxygen therapy can be applied in mild and moderate non-hypercapnia cases. A lung-saving ventilation strategy must be implemented in acute respiratory distress syndrome and mechanically ventilated patients. Extracorporeal membrane oxygenation is a highly specialized method, available only in selected centers and not applicable to a significant number of cases. Specific pharmacological treatment for COVID-19 is not currently available. Modern medicine is gearing up to fight the new coronavirus pandemic. The key is a holistic approach to the patient including, primarily, the use of personal protective equipment to reduce the risk of further virus transmission, as well as patient management, which consists in both quarantine and, in the absence of specific pharmacological therapy, symptomatic treatment. (Cardiol J 2020; 27, 2: 175-183)
\end{abstract}

Key words: coronavirus, COVID-19, SARS-CoV-2, pandemic, symptoms, epidemiology, healthcare, treatment, medical stuff

\section{Introduction}

Since the beginning of December 2019, there has been an epidemic of severe acute respiratory syndrome coronavirus 2 (SARS-CoV-2), which was originally referred to as $2019-\mathrm{nCoV}$. The first cases of infection and the subsequent development of the disease named COVID-19 were identified in the city of Wuhan, in the Chinese province of Hubei. On March 11, 2020, the World Health Organization (WHO) declared SARS-CoV-2 pandemic. The virus has now spread almost worldwide and as of March 31, 2020,

Address for correspondence: Lukasz Szarpak, Assoc. Prof. PhD, Lazarski University, ul. Świeradowska 43, 02-662 Warszawa, Poland, tel: +48 500186225, e-mail: lukasz.szarpak@gmail.com 
there have been 862,234 confirmed infection cases and 42,404 deaths.

The information on the coronavirus is updated daily. Currently, the epicenter is already outside China, in the United States of America (USA), Italy and Spain, where 189,633 and 105,792 and 95,923 cases, respectively, were confirmed as of March 31,2020 . The number of COVID-19 deaths in Italy has far exceeded the number of deaths recorded in China. Now it equals 8165 cases, while in China 3169 cases were reported. The new epicenter in the USA is prognosed with an increasing number of new cases emerging daily.

The aim of this article was to describe the taxonomy and pathogenicity of coronaviruses, including SARS-CoV-2, and to present recommendations for the diagnosis and management of patients with COVID-19.

\section{Epidemic versus pandemic}

An epidemic can be identified when a disease occurs at a certain time and in a certain area, and there are more cases than expected. A pandemic has a global scope: it involves several continents. Historically, one can point to several epidemics, including the plague of 1347-1351, which killed over 25 million people. The most recent recurrence of the plague took place between 1855 and 1859, when it was called the "third great plague pandemic" [1]. Smallpox, on the other hand, between 1520 and 1979, killed 56 million people. However, one of the deadliest epidemics was Spanish influenza, which killed over 50 million people between 1918 and 1920. During the epidemic, several waves of the disease were recorded. At first, it was highly contagious, but mild in its course; however, the second wave of the flu began in August and was characterized by extremely high mortality, mainly because of the additional occurrence of bronchial pneumonia [2]. The third phase took place in the winter of 1918/1919. A characteristic feature of the last two phases of the so-called Spanish influenza was a high mortality rate among 20-40-year-olds, which is unusual for "normal" seasonal influenza, which takes its lethal toll mainly on the youngest children and seniors. Other epidemics include the Asian influenza (1957-1958), which caused between 1 and 2 million deaths [3], the Hong Kong influenza (1968-1970), with 1-4 million deaths [4], the swine influenza (2009-2010), with 284,500 deaths [5], and the Ebola epidemic (2013-2016), with 11,300 deaths [6].

\section{History of coronavirus-related diseases}

Coronaviruses belong to the Coronoviridae family, classified in recent years in the order of Nidovirales [7]. Depending on the serotype and genotype, coronaviruses are divided into four genera, designated as $\alpha, \beta, \gamma$, and $\delta$ [8], with human coronaviruses $(\mathrm{HCoV})$ belonging to the first two [9].

Coronaviruses cause disease in animals and people around the world. $\mathrm{HCoV}$ is mainly known to cause infections of the upper and lower respiratory tract but the symptoms may also involve the nervous and digestive systems [10]. Of all the coronaviruses that threaten humans, 5 were described in the $21^{\text {st }}$ century. These are: HCoV-NL63, HCoV-HKU1, HCoV-SARS, HCoV-MERS and SARS-CoV-2. The first coronaviruses reported in 1966 and 1967, respectively — were HCoV-229E and $\mathrm{HCoV}-\mathrm{OC} 43$, both presented with cold symptoms. Initially, only these two coronaviruses were considered to be dangerous for patients. In 2003, a breakthrough occurred, and $\mathrm{HCoV}$-SARS virus was identified. Both HCoV-SARS and HCoV-MERS are zoonotic viruses, causing human infection from animals. It is assumed that bats are their natural reservoir (Fig. 1) [11].

In the case of zoonotic viruses, the interspecies barrier between bats and other mammals has been broken and, as a result of the fixation of the virus's transmission in these animal groups, the animals have become an intermediate link. The only other element was the transmission of the infection to humans. It can therefore be concluded that the interspecies barrier was broken down at least twice to make such pathogens as HCoV-SARS and $\mathrm{HCoV}$-MERS dangerous to humans.

HCoV-SARS virus was responsible for more than 8000 cases in people between 2002 and 2003, mainly in Asia, and was the etiological agent of severe acute respiratory syndrome (SARS). At that time, over 700 cases ended in the death of those people. In the transmission chain of the $\mathrm{HCoV}$ -SARS virus infection, Asian palm civet played a major role, which is considered a delicacy in Chinese cuisine. Another indirect host for HCoV-SARS was the Asian yenote $[12,13]$. The HCoV-SARS virus is transmitted by the droplet pathway, through a close contact with an infected person. The transmission in aerosol form in ventilation systems of buildings has also been confirmed. The virus enters the lungs, where it directly damages alveoli cells causing acute respiratory failure. The incubation 


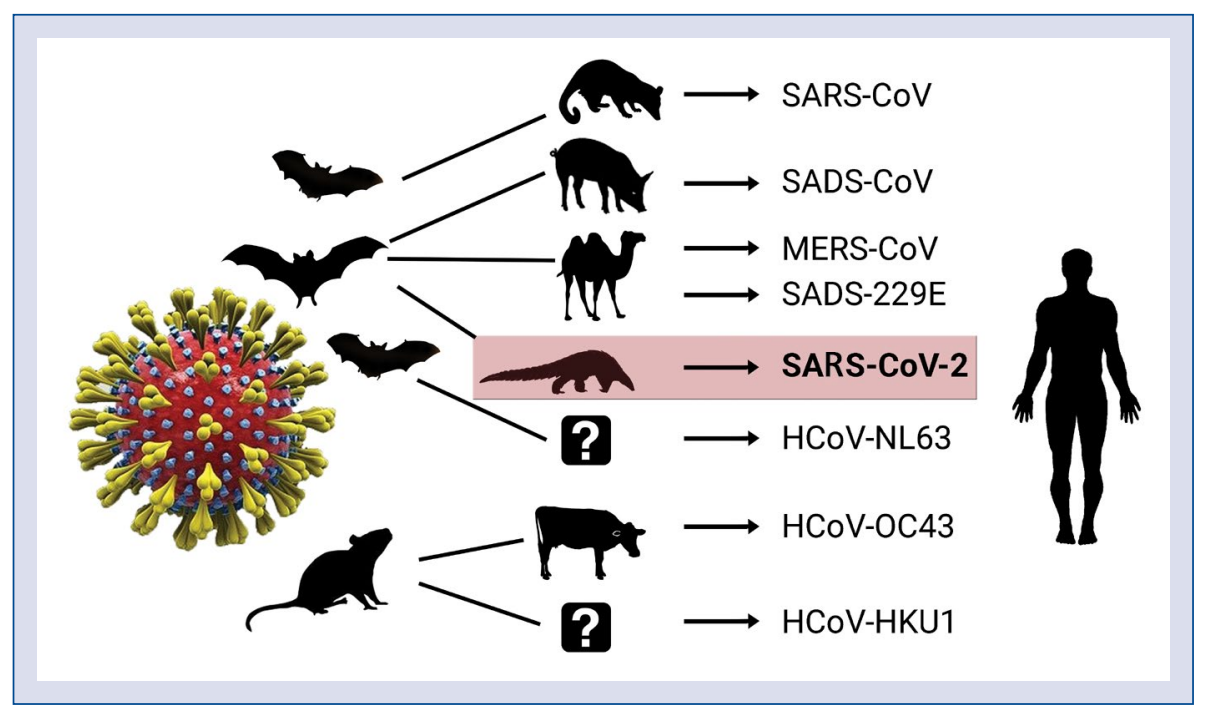

Figure 1. Animal origin of human coronaviruses.

period for symptoms of SARS virus infection is 3-7 days [14]. The initial phase of the disease does not present specifically; flu-like symptoms dominate: fever with chills, fatigue, headaches, muscle pain, malaise, lack of appetite, while nausea, vomiting, rhinitis, coughing, or dizziness are less frequent. These symptoms may persist for 3-7 days, followed by rapid decompensation of the patient as a result of inflammation of the lower airways, manifested by dry cough, dyspnea, and respiratory failure. Mortality as a result of HCoV-SARS is estimated at $7 \%$.

For the HCoV-MERS virus, causing Middle East respiratory syndrome (MERS) - an acute infectious disease - camels are the indirect host. Seroepidemic studies have shown that the HCoV-MERS-like virus has been causing camel infections and diseases for years, especially in the Arabian Peninsula and Africa. Human infection can also occur through contact with excretions of an infected camel (from the respiratory tract, urine, feces, etc.), as well as during the processing of camel meat and other products.

The disease is characterized by fever above 38 degrees Celsius, cough, and other respiratory symptoms, such as shortness of breath and dyspnea; moreover, pneumonia, acute respiratory failure, septic shock, or multi-organ failure may develop. Gastrointestinal symptoms (diarrhea) are sometimes observed. Death occurs in over $36 \%$ of patients. The hatching period ranges from 2 to 14 days. There is no vaccine for MERS and the treatment is symptomatic $[14,15]$. Because of a high resistance of coronaviruses to environmental conditions and the ability to maintain infectivity in the form of aerosol, it is believed that the disease is most likely to occur in the droplet pathway.

SARS-CoV-2 and COVID-19: Epidemiology, pathology, and symptoms

The first case of SARS-CoV-2 infection in the form of acute respiratory failure was reported on December 12, 2019 in the city of Wuhan in the central-eastern province of China, Hubei. The symptomatology resembled that of SARS [16]. Scientists immediately started to investigate the new coronavirus, and the first genome of COVID-19 was published by a research team led by Prof. Zhang on January 10, 2020 [17]. The SARS-CoV-2 genotype resembles that of viruses causing SARS (45-90\%) and MERS (20-60\%). The name of the virus refers to the fact that it causes airway infections similar to SARS. However, SARS-CoV-2 presents the greatest genetic similarity, of nearly $89 \%$, to the coronavirus genome found in bats [18]. This may indicate that bats were the first host. On the other hand, Lorusso et al. [19] observed 99\% identical coronavirus strains in Chinese civets. Subsequent cases in which SARS and MERS were excluded were reported on December 31, 2019, while the determination of the infection causative factor was made public 9 days later [20]. Due to the onset of the pandemic in China, most scientific publications on the virus currently refer to Chinese patients. As is the case of SARS and MERS, the primary route of spreading COVID-19 is a droplet pathway. 


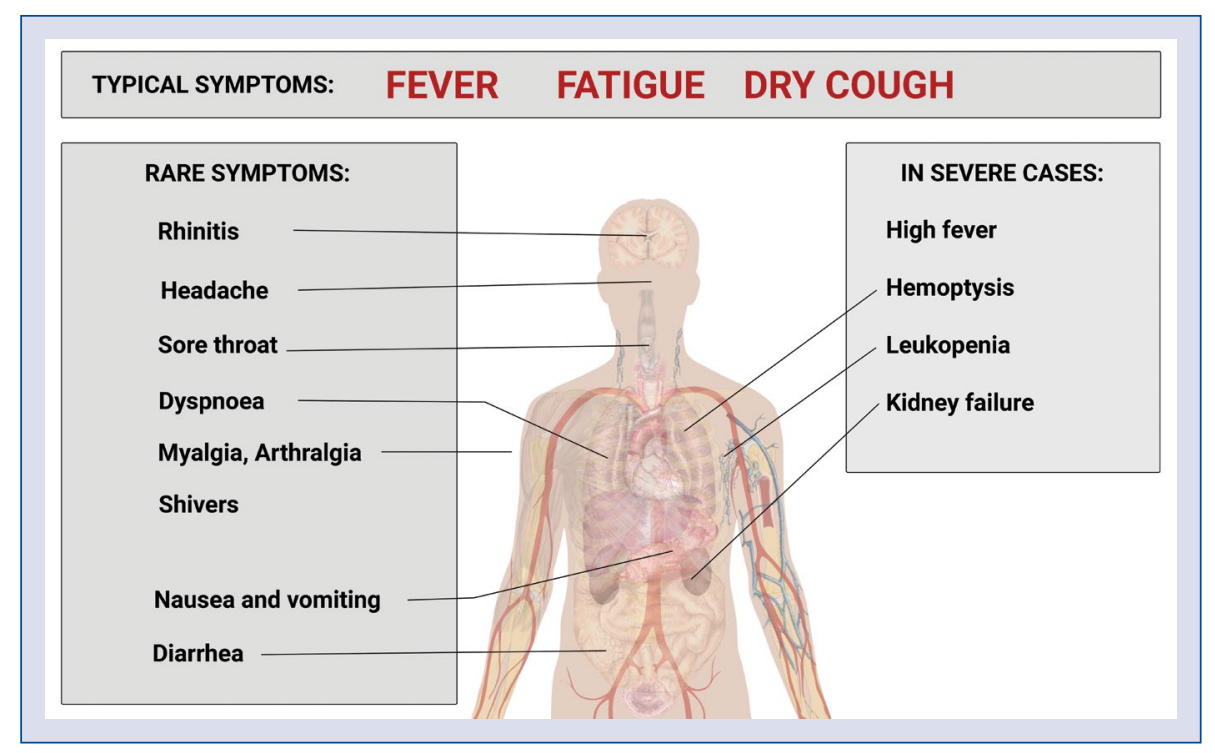

Figure 2. COVID-19 symptoms.

It is worth remembering that HCoV-SARS, HCoV-MERS, and SARS-CoV-2 can also be transmitted via environmental vectors. Wang et al. [21] indicated that the time from the onset of the disease to death was shorter in people over 70 years of age compared to younger patients (11.5 and 20 days, respectively). Yang et al. [22] indicated that in COVID-19-infected patients, comorbidities and the diagnosed underlying diseases, including hypertension, respiratory system and cardiovascular diseases, which may be risk factors for severe when compared with a non-severe course of the disease.

The complete clinical manifestation is not clear yet, as the reported symptoms range from mild to severe, with some cases resulting in death. Guan et al. [23] have shown that the most common symptoms are fever (in $88.7 \%$ of patients), cough $(67.8 \%)$, fatigue (38.1\%), sputum production (33.7\%), shortness of breath (18.7\%), myalgia or arthralgia (14.9\%), or sore throat (13.9\%). In addition, symptoms from the nervous and digestive systems should also be considered. Liu et al. [24] also indicate headache $(53.3 \%)$ as an important symptom of the disease, as well as nausea, vomiting, or diarrhea (30\%). Figure 2 shows both typical and less frequent symptoms of COVID-19. In many, the infection remains asymptomatic. Li et al. [25] implied that patients with mild symptoms were reported to recover after 1 week while severe cases experienced progressive respiratory failure due to alveolar damage from the immunological response to the virus, which may lead to death. Severely affected patients are referred to intensive care units because of acute pneumonia, sepsis, septic shock, or acute respiratory distress syndrome.

The hatching period for COVID-19 lasts 2-14 days, but current studies indicate that it can be as long as 21 days [18, 26]. The virus is highly infectious, as confirmed by recent epidemiological data. As of March 26, 2020, the reported number of confirmed infection cases equaled 510,108. COVID-19 mortality is $4.5 \%$ and turns out to be lower than that in SARS or MERS, but the disease dynamics are very high.

\section{COVID-19 suspicion procedure}

The best prevention is to avoid virus exposure. In addition, contact persons should be subjected to mandatory quarantine. However, this is not possible for medical personnel, who should therefore be equipped with specialized personal protective equipment. Airborne precautions and other protective measures have been discussed and proposed for prevention [27]. Proper use and disposal of masks is important to avoid any increase in risk of transmission [28, 29]. Infection preventive and control measures that may reduce the risk of exposure include the following: use of face masks; covering coughs and sneezes with tissues that are then safely disposed of; regular hand washing with soap or disinfection with hand sanitizer containing at least $60 \%$ alcohol; avoidance of contact with infected people and maintaining an appropriate distance; and refraining from touching eyes, nose, and mouth with unwashed hands. 


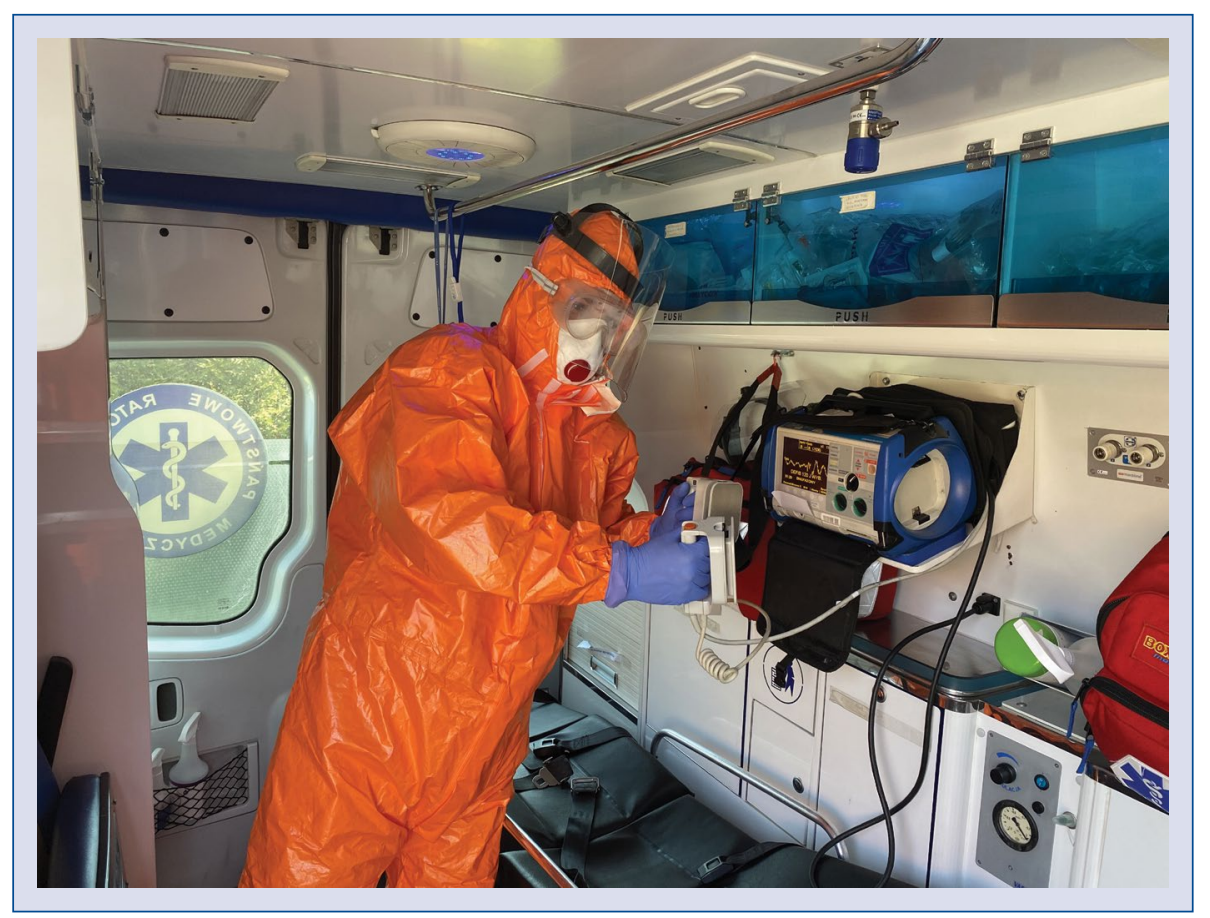

Figure 3. Paramedic dressed in antibiological protection equipment.

While the infected persons should wear masks to reduce the risk of infecting other people, medical personnel should be equipped with particulate respirators such as the certified N95 or FFP2 when performing aerosol-generating procedures and use of medical masks while providing any care to suspected or confirmed cases [30]. In accordance with WHO guidelines on the use of masks in the community, during home care and in health care settings in the context of the novel coronavirus 2019-nCoV [29], persons with respiratory symptoms should wear medical masks in health care and home care settings, properly following the infection prevention guidelines. The individuals without respiratory symptoms are not required to wear medical masks when in public, although this statement is questioned by an increasing number of experts. Medical personnel, including paramedics, in addition to protective masks of N95 or FFP2 type, should also be equipped with antibiological protection, including specialized overalls, gloves, glasses, or visors (Fig. 3).

When a SARS-CoV-2 infection is suspected, emergency teams as well as medical dispatchers should apply specific algorithms of management. An example is presented in Figure 4. The management can be divided into two main paths, depending on the patient's condition. If symptoms of upper respiratory tract infection are observed without dyspnea and with temperatures below 38 degrees Celsius, the patient should be directed to an isolation site and await test result. If the patient's condition deteriorates, a dedicated ambulance should transport them to an infectious department or dedicated infectious hospital. These departments or hospitals should also be chosen for patients with dyspnea, cough, and temperatures above 38 degrees Celsius, including those in severe conditions with coexisting diseases requiring intensive medical monitoring.

Transporting a patient with a suspicion of COVID-19 should always be performed by a dedicated emergency team. However, in many situations, the emergency medical service staff perform interventions where they may suspect a coronavirus infection only on the basis of an examination and interview. Then, after transport, the ambulance should be disinfected with disinfectants with a wide range of viricidal effects [26].

\section{Diagnosis}

The main step in the diagnosis of SARS-CoV-2 infection is positive epidemiological history. Deng and Peng [31] indicate that risk factors should be divided into two groups: epidemiological (group A) and clinical ones (group B). If any group A factor occurs, once at least two group B symptoms are 


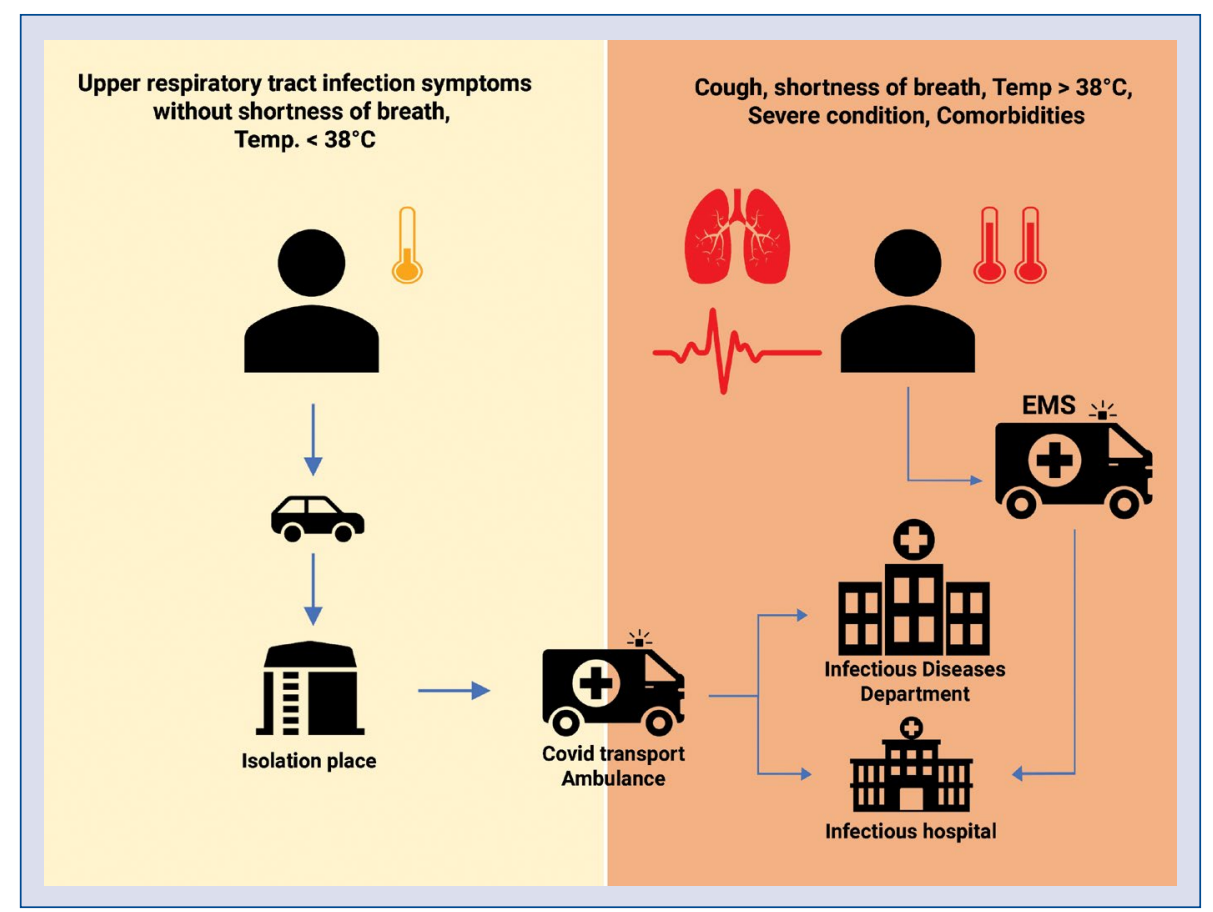

Figure 4. Algorithm for dealing with suspected SARS-CoV-2 infection.

present, the infection should be suspected. Epidemiological factors include: traveling to or living in an area where the disease occurs; contact with a patient who has a confirmed infection; contact with a patient with fever and respiratory symptoms from an area where the infection occurs; occurrence of the disease in the group/family. Clinical factors are the following: fever and/or respiratory symptoms; pneumonia with radiological symptoms; normal or reduced white blood cell count or reduced lymphocyte count in the early stages of the disease. However, first stage cases may be asymptomatic, with no fever. A confirmed case is a suspected case with a positive molecular test. Specific diagnosis is performed by specific molecular tests with respiratory samples (throat swab, nasopharyngeal swab, sputum, endotracheal aspirate, bronchoalveolar lavage). The virus may also be detected in the stool and, in severe cases, blood. It must be remembered that the multiplex polymerase chain reaction (PCR) panels currently available do not include COVID-19.

All patients with severe COVID-19 should be screened for hyperinflammation by using laboratory trends. Lymphopenia and leukopenia are characteristic of COVID-19 in its initial stage; however, as indicated by Lippi and Plebani [32], about 30\% of patients treated in China were found to have increased leukocyte values. In infected patients, increased myoglobin levels, as well as increased creatine kinase, aminotransferases (ALT and AST) and lactate dehydrogenase activity are also found. $\mathrm{C}$-reactive protein concentration presents elevated values in these patients with normal procalcitonin levels. With severe infection, an increase in creatinine, D-dimers, and lactate levels can also be observed [33]. A cytokine profile resembling secondary hemophagocytic lymphohistiocytosis is associated with COVID-19 severity, characterized by increased interleukin MCP1, MIP1a, IL-2, IL-7, IL-10, GSCF, IP10, or TNF- $\alpha$ [34, 35].

In the case of SARS-CoV-2 infection, because of the affinity of the virus to the airways, it is important to perform a chest X-ray. Computed tomography scanning provides an important basis for early diagnosis and treatment of novel coronavirus pneumonia. In the course of COVID-19, one can expect changes characteristic of severe respiratory tract infections similar to those in SARS. In the initial stage of infection, interstitial lesions and patchy ground glass opacities in the peripheral areas under the pleura dominate the radiological picture. In the next phase, these areas are rounded off and infiltrating changes appear. However, among severe patients, characteristic consolidation changes without pleural effusion can be observed [36].

For patients with suspected infection, the following diagnostic procedures have been suggested: 
performing real-time fluorescence (RT-PCR) to detect the positive nucleic acid of SARS-CoV-2 in sputum, throat swabs, and secretions of the lower respiratory tract samples [37].

\section{Treatment}

COVID-19 patients should be treated in specialist centers. The current trend is to create unified infectious hospitals in which patients with confirmed SARS-CoV-2 infection are treated. A significant number of patients with pneumonia require passive oxygen therapy. In the first phase of oxygen therapy, a flow of $5 \mathrm{~L} / \mathrm{min}$ is applied and then gradually titrated to obtain an oxygen concentration of $\geq 90 \% \mathrm{SpO}_{2}$ for adults and children and 92-95\% $\mathrm{SpO}_{2}$ for pregnant women. As indicated by Huang et al. [35], $5 \%$ of patients requiring invasive mechanical ventilation. These are mainly patients with acute respiratory distress syndrome and hypoxemic respiratory failure.

Non-invasive ventilation and high-flow nasal oxygen therapy can be applied in mild and moderate non-hypercapnia cases, but the patient should be assessed for respiratory failure. Li [38] suggests that if there is no improvement within 1 hour despite high-flow nasal oxygen, endotracheal intubation and mechanical lung ventilation should be used. A lung-saving ventilation strategy must be applied. The respiratory rate should be as low as possible and the tidal volume should not exceed $6 \mathrm{~mL}$ for predicted body weight to maintain a $\mathrm{pH}$ greater than 7.2. During mechanical ventilation, positive end-expiratory pressure should also be used. For the most critically ill patients, extracorporeal membrane oxygenation is an option [39]. However, it should be stressed that this is a highly specialized method, available only in selected centers and not applicable to a significant number of cases.

Specific pharmacological treatment for COVID-19 is not currently available. Many centers use empirical antiviral treatment with lopinavir/ritonavir, remdesivir, ribavirin, nelfinavir, umifenovir, favipiravir, or other derivatives, among others [40, 41]. Camostat mesylate, licensed in pancreatitis and reflux esophagitis in Japan is postulated to inhibit a human protein involved with infection - it is a commercially available inhibitor of the enzyme transmembrane protease, serine 2 (TMPRSS2) [42]. Anti-malaria drugs are postulated. Among them, chloroquine is registered as the drug for mild stages of COVID-19 in few countries [43], off label use of its derivative - hydroxychloroquine is ad- vised by some groups of experts [44]. Therapeutic options also include steroids, intravenous immunoglobulin, selective cytokine blockade (e.g. anakinra or tocilizumab), and Janus kinase inhibition (baricitinib) [45]. Antibiotics should not be applied without microbiological confirmation of bacterial infection, with an exception of chloroquine/hydroxychloroquine combined with azithromycin as an experimental way to reduce the viral load $[46,47]$. In cases of infection with a bacterial background, broad-spectrum antibiotics are recommended. In septic shock, glucocorticosteroids should be administered, e.g. hydrocortisone at an intravenous dose of $50 \mathrm{mg}$ repeated every 6 hours, which is, however, subject to discussion. In patients with circulatory failure, norepinephrine should be applied to maintain organ perfusion while reducing fluid supply. The first non-blinded partially randomized trial organized by the WHO - SOLIDARITY trial — which compares 4 types of treatment: remdesivir vs. chloroquine/hydroxychloroquine vs. lopinavir/ritonavir vs. lopinavir/ ritonavir plus interferon-beta were launched on March, $20^{\text {th }}, 2020$. WHO's website will randomize patients to local standard care or one of the four drug regimens, using the only ones available at the patient's hospital. Some simple outcomes, which are easy to collect and report will be gathered: the day the patient left hospital or died, the duration of hospital stay, requirements of oxygen or ventilation [48, 49]. It might reveal some real evidence for COVID-19 treatment until new methods, vaccines, convalescent plasma products, specific monoclonal antibodies directed at SARS-CoV-2 or a sudden stop of pandemic is awaited.

\section{Summary}

Modern medicine, including emergency medicine, is geared to fighting the new coronavirus. Since the appearance of the first cases in December 2019, SARS-CoV-2 has been the cause of the pandemic. The key is a holistic approach to the patient, primarily including the use of personal protective equipment to reduce the risk of further virus transmission, as well as patient management, which consists in both quarantine and, in the absence of proved specific pharmacological therapy, symptomatic treatment.

\section{Acknowledgements}

Study was supported by the ERC Research Net as well as the Polish Society of Disaster Medicine.

Conflict of interest: None declared 


\section{References}

1. Bramanti B, Dean KR, Walløe L, et al. The Third Plague Pandemic in Europe. Proc Biol Sci. 2019; 286(1901): 20182429, doi: 10.1098/rspb.2018.2429, indexed in Pubmed: 30991930.

2. Flecknoe D, Wakefield BC, Simmons A. Plagues \& wars: the 'Spanish Flu' pandemic as a lesson from history. Med Confl Surviv. 2018; 34(2): 61-68, doi: 10.1080/13623699.2018.1472892.

3. Joseph U, Linster M, Suzuki Y, et al. Adaptation of pandemic H2N2 influenza A viruses in humans. J Virol. 2015; 89(4): 2442-2447, doi: 10.1128/JVI.02590-14, indexed in Pubmed: 25505070.

4. Viboud C, Grais RF, Lafont BAP, et al. Multinational impact of the 1968 Hong Kong influenza pandemic: evidence for a smoldering pandemic. J Infect Dis. 2005; 192(2): 233-248, doi: 10.1086/431150, indexed in Pubmed: 15962218.

5. Li H, Cao B. Pandemic and avian influenza a viruses in humans: epidemiology, virology, clinical characteristics, and treatment strategy. Clin Chest Med. 2017; 38(1): 59-70, doi: 10.1016/j. ccm.2016.11.005, indexed in Pubmed: 28159162.

6. Baseler L, Chertow DS, Johnson KM, et al. The pathogenesis of ebola virus disease. Annu Rev Pathol. 2017; 12: 387-418, doi: 10.1146/ annurev-pathol-052016-100506, indexed in Pubmed: 27959626.

7. Adams MJ, Carstens EB. Ratification vote on taxonomic proposals to the International Committee on Taxonomy of Viruses (2012). Arch Virol. 2012; 157(7): 1411-1422, doi: 10.1007/ /s00705-012-1299-6, indexed in Pubmed: 22481600.

8. Wong ACP, Li X, Lau SKP, et al. Global epidemiology of bat coronaviruses. Viruses. 2019; 11(2): E174, doi: 10.3390/v11020174, indexed in Pubmed: 30791586.

9. Hu B, Ge X, Wang LF, et al. Bat origin of human coronaviruses. Virol J. 2015; 12: 221, doi: 10.1186/s12985-015-0422-1, indexed in Pubmed: 26689940.

10. Adhikari SP, Meng S, Wu YJ, et al. Epidemiology, causes, clinical manifestation and diagnosis, prevention and control of coronavirus disease (COVID-19) during the early outbreak period: a scoping review. Infect Dis Poverty. 2020; 9(1): 29, doi: 10.1186/ s40249-020-00646-x, indexed in Pubmed: 32183901.

11. Banerjee A, Kulcsar K, Misra V, et al. Bats and Coronaviruses. Viruses. 2019; 11(1), doi: 10.3390/v11010041, indexed in Pubmed: 30634396.

12. Cui J, Li F, Shi ZL. Origin and evolution of pathogenic coronaviruses. Nat Rev Microbiol. 2019; 17(3): 181-192, doi: 10.1038/ /s41579-018-0118-9, indexed in Pubmed: 30531947.

13. Luk HKH, Li X, Fung J, et al. Molecular epidemiology, evolution and phylogeny of SARS coronavirus. Infect Genet Evol. 2019; 71: 21-30, doi: 10.1016/j.meegid.2019.03.001, indexed in Pubmed: 30844511.

14. Bradley BT, Bryan A. Emerging respiratory infections: the infectious disease pathology of SARS, MERS, pandemic influenza, and Legionella. Semin Diagn Pathol. 2019; 36(3): 152-159, doi: 10.1053/j.semdp.2019.04.006, indexed in Pubmed: 31054790.

15. Singh SK. Middle east respiratory syndrome virus pathogenesis. Semin Respir Crit Care Med. 2016; 37(4): 572-577, doi: 10.1055/s-0036-1584796, indexed in Pubmed: 27486737.

16. Peeri NC, Shrestha N, Rahman MdS, et al. The SARS, MERS and novel coronavirus (COVID-19) epidemics, the newest and biggest global health threats: what lessons have we learned? Int J Epidemiol. 2020 [Epub ahead of print], doi: 10.1093/ije/dyaa033, indexed in Pubmed: 32086938.

17. Malik YS, Sircar S, Bhat S, et al. Emerging novel coronavirus (2019-nCoV)-current scenario, evolutionary perspective based on genome analysis and recent developments. Vet Q. 2020; 40(1): 68-76, doi: 10.1080/01652176.2020.1727993, indexed in Pubmed: 32036774.

18. Lai CC, Shih TP, Ko WC, et al. Severe acute respiratory syndrome coronavirus 2 (SARS-CoV-2) and coronavirus disease-2019 (COVID-19): the epidemic and the challenges. Int J Antimicrob Agents. 2020; 55(3): 105924, doi: 10.1016/j.jjantimicag.2020.105924, indexed in Pubmed: 32081636.

19. Lorusso A, Calistri P, Petrini A, et al. Novel coronavirus (SARSCoV-2) epidemic: a veterinary perspective. Vet Ital. 2020 [Epub ahead of print], doi: 10.12834/VetIt.2173.11599.1, indexed in Pubmed: 32048818.

20. Wu F, Zhao Su, Yu B, et al. A new coronavirus associated with human respiratory disease in China. Nature. 2020; 579(7798): 265-269, doi: 10.1038/s41586-020-2008-3, indexed in Pubmed: 32015508 .

21. Wang W, Tang J, Wei F. Updated understanding of the outbreak of 2019 novel coronavirus (2019-nCoV) in Wuhan, China. J Med Virol. 2020; 92(4): 441-447, doi: 10.1002/jmv.25689, indexed in Pubmed: 31994742.

22. Yang J, Zheng Ya, Gou Xi, et al. Prevalence of comorbidities in the novel Wuhan coronavirus (COVID-19) infection: a systematic review and meta-analysis. Int J Infect Dis. 2020 [Epub ahead of print], doi: 10.1016/j.ijid.2020.03.017, indexed in Pubmed: 32173574.

23. Guan WJ, Ni ZY, Hu Yu, et al. Clinical characteristics of coronavirus disease 2019 in China. N Engl J Med. 2020 [Epub ahead of print], doi: 10.1056/NEJMoa2002032, indexed in Pubmed: 32109013.

24. Liu M, He P, Liu HG, et al. [Clinical characteristics of 30 medical workers infected with new coronavirus pneumonia]. Zhonghua Jie He He Hu Xi Za Zhi. 2020; 43(3): 209-214, doi: 10.3760/cm a.j.issn.1001-0939.2020.03.014, indexed in Pubmed: 32164090.

25. Li T, Wei C, Li W, et al. Beijing Union Medical College Hospital on "pneumonia of novel coronavirus infection" diagnosis and treatment proposal (V2.0). Med J Peking Union Med Coll Hosp. 2020.

26. Yen MY, Schwartz J, Chen SY, et al. Interrupting COVID-19 transmission by implementing enhanced traffic control bundling: Implications for global prevention and control efforts. J Microbiol Immunol Infect. 2020 [Epub ahead of print], doi: 10.1016/j. jmii.2020.03.011, indexed in Pubmed: 32205090.

27. Smereka J, Szarpak L, Filipiak KJ. Modern medicine in COVID-19 era. Disaster Emerg Med J. 2020, doi: 10.5603/DEMJ.a2020. 0012.

28. Szarpak L, Smereka J, Filipiak KJ, et al. Cloth masks versus medical masks for COVID-19 protection. Cardiol J. 2020; 27(2): 218-219, doi: 10.5603/CJ.a2020.0054.

29. WHO. Advice on the use of masks in the community, during home care and in health care settings in the context of the novel coronavirus 2019-nCoV outbreak (interim guidance). 2020. WHO/nCov/IPC_Masks/2020. Accessed 3 Feb 2020.

30. Wax R, Christian M. Practical recommendations for critical care and anesthesiology teams caring for novel coronavirus $(2019-\mathrm{nCoV})$ patients. Can J Anaesth. 2020, doi: 10.1007/s12630-020-01591-x.

31. Deng SQ, Peng HJ. Characteristics of and public health responses to the coronavirus disease 2019 outbreak in China. J Clin Med. 2020; 9(2), doi: 10.3390/jcm9020575, indexed in Pubmed: 32093211.

32. Lippi G, Plebani M. Laboratory abnormalities in patients with COVID-2019 infection. Clin Chem Lab Med. 2020 [Epub ahead 
of print], doi: 10.1515/cclm-2020-0198, indexed in Pubmed: 32119647.

33. Rodriguez-Morales AJ, Cardona-Ospina JA, Gutiérrez-Ocampo E, et al. Clinical, laboratory and imaging features of COVID-19: a systematic review and meta-analysis. Travel Med Infect Dis. 2020 [Epub ahead of print]: 101623, doi: 10.1016/j. tmaid.2020.101623, indexed in Pubmed: 32179124.

34. Mehta P, McAuley DF, Brown M, et al. COVID-19: consider cytokine storm syndromes and immunosuppression. Lancet. 2020; 395(10229): 1033-1034, doi: 10.1016/S0140-6736(20)30628-0, indexed in Pubmed: 32192578.

35. Huang C, Wang Y, Li X, et al. Clinical features of patients infected with 2019 novel coronavirus in Wuhan, China. Lancet. 2020; 395(10223): 497-506, doi: 10.1016/s0140-6736(20)30183-5.

36. Xu YH, Dong JH, An WM, et al. Clinical and computed tomographic imaging features of novel coronavirus pneumonia caused by SARS-CoV-2. J Infect. 2020; 80(4): 394-400, doi: 10.1016/j. jinf.2020.02.017, indexed in Pubmed: 32109443.

37. Chen $\mathrm{W}, \mathrm{Xu} Z, \mathrm{Mu}$ J, et al. Real-time quantitative fluorescent reverse transcriptase-PCR for detection of severe acute respiratory syndrome-associated coronavirus RNA. Mol Diagn. 2004; 8(4): 231-235, doi: 10.1007/bf03260067, indexed in Pubmed: 15887978.

38. Li T. Diagnosis and clinical management of severe acute respiratory syndrome coronavirus 2 (SARS-CoV-2) infection: an operational recommendation of Peking Union Medical College Hospital (V2.0). Emerg Microbes Infect. 2020; 9(1): 582-585, doi: 10.1080/22221751.2020.1735265, indexed in Pubmed: 32172669.

39. MacLaren G, Fisher D, Brodie D. Preparing for the most critically ill patients with COVID-19: the potential role of extracorporeal membrane oxygenation. JAMA. 2020 [Epub ahead of print], doi: 10.1001/jama.2020.2342, indexed in Pubmed: 32074258.

40. Zhang L, Liu Y. Potential interventions for novel coronavirus in China: a systematic review. J Med Virol. 2020; 92(5): 479-490, doi: 10.1002/jmv.25707, indexed in Pubmed: 32052466.
41. Shen LiW, Mao HJ, Wu YL, et al. TMPRSS2: a potential target for treatment of influenza virus and coronavirus infections. Biochimie. 2017; 142: 1-10, doi: 10.1016/j.biochi.2017.07.016, indexed in Pubmed: 28778717.

42. Yamaya M, Shimotai Y, Hatachi Y, et al. The serine protease inhibitor camostat inhibits influenza virus replication and cytokine production in primary cultures of human tracheal epithelial cells. Pulm Pharmacol Ther. 2015; 33: 66-74, doi: 10.1016/j. pupt.2015.07.001, indexed in Pubmed: 26166259.

43. Polish Registration on March, 13th, 2020. http://www.urpl.gov. pl/sites/default/files/Arechin\%20Ulotka\%20dla\%20Pacjenta.pdf.

44. Cortegiani A, Ingoglia G, Ippolito M, et al. A systematic review on the efficacy and safety of chloroquine for the treatment of COVID-19. J Crit Care. 2020 [Epub ahead of print], doi: 10.1016/j. jcrc.2020.03.005, indexed in Pubmed: 32173110.

45. Richardson P, Griffin I, Tucker C, et al. Baricitinib as potential treatment for 2019-nCoV acute respiratory disease. Lancet. 2020; 395(10223): e30-e31, doi: 10.1016/s0140-6736(20)30304-4.

46. Ruetzler K, Szarpak L, Filipiak KJ, et al. The COVID-19 pandemic - a view of the current state of the problem. Disaster Emerg Med J. 2020, doi: 10.5603/DEMJ.a2020.0015.

47. Gautret P, Lagier JC, Parola P, et al. Hydroxychloroquine and azithromycin as a treatment of COVID-19: results of an openlabel non-randomized clinical trial. Int J Antimicrob Agents. 2020 [Epub ahead of print]: 105949, doi: 10.1016/j.ijantimicag.2020.105949, indexed in Pubmed: 32205204.

48. Kupferschmidt K, Cohen J. Race to find COVID-19 treatments accelerates. Science. 2020; 367(6485): 1412-1413, doi: 10.1126/ /science.367.6485.1412, indexed in Pubmed: 32217705.

49. Smereka J, Szarpak L. COVID 19 a challenge for emergency medicine and every health care professional. Am J Emerg Med. 2020 [Epub ahead of print], doi: 10.1016/j.ajem.2020.03.038, indexed in Pubmed: 32241630. 